\title{
29. COMMISSION DES SPECTRES STELLAIRES
}

\section{Reports of Meetings}

President: L. H. Aller. SECRETARY: M. Hack.

\section{Business Meeting, 26 August 1964}

The agenda is the following:

(I) Corrections to the Draft Report

(2) Changes in membership

(3) New officers

(4) Standards of Line Intensities

(5) HD Catalogue republication

(6) Stellar Spectra Atlas

(I) The President asked that corrections to the Draft Report be reported to him as soon as possible.

(2) New members to the Commission were proposed and elected to membership. It was pointed out that a new Commission was being established for spectral classification. Those primarily interested in this field should transfer to Commission 45 .

The assembly observed one minute of silence in memory of Otto Struve.

(3) The following astronomers were proposed as new officers of Commission 29:

President: G. Sahade

Vice-President: M. W. Feast

Organizing Committee: Fujita, Boyarchuk, Wright, Y. Andrillat, Oke.

Their election was unanimously approved by the Commission.

(4) K. O. Wright reported on Standards of Spectral Line Intensities. A list of lines for I 2 main sequence stars Bo to $\mathrm{G}_{5}$, spectral range $3900-4500 \AA$ will be published (Publ. Dom. astrophys. Obs., 12, no. 7). The dispersion is always greater than $10 \AA / \mathrm{mm}$.

The Victoria observations are $+5 \%$

The Mt Wilson " , $\quad-6 \%$

The Mt Palomar $\quad, \quad, \quad-10 \%\}$ compared with the average

The 'average dispersion' spectrograms $(3 \circ \AA / \mathrm{mm})+15 \%$

Dr K. O. Wright proposed Mme G. Cayrel de Strobel as chairman for the next working committee.

(5) The need for a revision or a republication of the HD Catalogue was discussed. A proper revision would be a very big undertaking, while a republication with a correction of known errors could be more easily done.

(6) Spectra of standard stars mainly taken at Mt Wilson and Mt Palomar Observatories are available. The problem is how to print all this material. Proposals were made to reproduce the spectra on glass, or to reproduce the tracings on microfilms. Further suggestions by people interested in this Atlas should be sent to Miss Underhill. 


\section{Scientific Meeting, 28 August 1964 \\ I. NASA PROGRAMS OF SPECTROSCOPIC OBSERVATIONS \\ FROM ROCKETS AND SATELLITES}

\section{N. Roman}

Rocket program: continuation of Stecher's program of obtaining spectra of stars in the region I $100 \AA-3500 \AA$ with resolution of 100 and $50 \AA$, by means of objective grating spectrographs. Also photographic spectra are obtained in the region $1600-3000 \AA$ with $20 \AA$ resolution.

Instrumentation for high resolution spectra (photographic) is constructed at Princeton Observatory for the range $1250-3000 \AA$, with a resolution of 1 or $2 \AA$. This is the best which can be done by rockets. Photoelectrically: A scanning spectrometer, $5 \AA$ resolution, is used with the 13 -inch telescope. The Princeton spectrometer has a resolution of $2 \AA$ and a spectral range of $900-3000 \AA$.

Satellite programs: OSO B2. Hallam is developing the objective grating technique. Spectral range: $1500-3000 \AA$ with $180 \AA$ resolution. B stars brighter than $6^{m}$ can be observed. The errors in the observed fluxes are $\pm 2 \%$ for $m=\mathrm{I} \cdot 5$

$$
\pm 10 \% \text { for } m=6.0
$$

The faint end is limited by both background and photon statistics. Other spectrometers

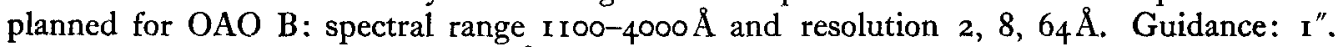
Six phototubes, approximately $600 \AA$ apart scan the whole spectrum. It is hoped to reach I $^{m}$ for B stars (and possibly $12^{m}$ ).

OAO C: a $3^{2-i n c h ~ m i r r o r ~ t e l e s c o p e . ~ T h e ~ s p e c t r o m e t e r ~ c o v e r s ~ t h e ~ s p e c t r a l ~ r a n g e ~ o f ~}$ $85^{\circ}-3300 \AA$. The slit is $0.1 \AA$ and $0.4 \AA$ wide. There are two carriages each for first and second order images plus two monitor detectors at ${ }_{13} 10 \AA$ and 343 I $\AA$ (2 $\AA$ wide) and also a $543 \AA$ detector to monitor scattered light.

Future plans: to invite guest observing plans and guest observing telescopes. However, this program will be realized only when all the equipment has been fully calibrated, checked out, and is performing in a satisfactory way. Plans for developing an infra-red telescope, an advanced orbiting astronomical observatory (AOAO) and MOT.

\section{Discussion}

Bidelman: Is there any prospect of photographic recording for satellite spectroscopy?

Miss Roman: We do not expect to be able to recover film from satellites in the near future. Hopefully, we may be able to recover a limited amount of film in the $1970-80$ period.

Gratton: What information can be given about the time necessary for an observation of a star of given magnitude and spectral type, with a certain spectral purity and a certain degree of accuracy? And are there considered any special devices for identifying the objects observed in the case of fields containing various stars within a small angular distance from each other?

Miss Roman: The stabilized rockets and the satellites have either a gyroscopic or a celestial reference system which permits stellar identification of stars in a cluster but no provision has been made for selecting components of double stars or stars in a dense cluster.

Henize: In reference to your answer to Dr Bidelman's question: Since there appears to be considerable advantage in using photography in initial survey work, is the possibility of carrying a moderate-sized astronomical camera on the Apollo flights completely ruled out?

Miss Roman: No.

Bidelman: You mentioned the possibility of a very large orbiting telescope. What is the 
prospect of such a telescope on the Moon? It seems to me fantastic that we can send a man to the Moon but cannot obtain a photograph of the ultraviolet spectrum of a star. It appears that this obviously important type of observation has a far lower priority than it deserves.

\section{REPORT OF THE RESULTS OF STRATOSCOPE II}

\section{Schwarzschild}

A 36 -inch telescope of almost conventional type and operated from the ground has been sent to an altitude of $25 \mathrm{~km}$ by means of helium balloons. This report concerns only the results of the second flight, that of November 1963. The movements and pointing of the telescope was commanded by means of television. Infra-red spectrometry was done of several stars. The resolution is $I / I 0$ of a micron $=1000 \AA$ and the spectral range is from $I$ to 3 microns. The spectrometer was calibrated in sensitivity using Sirius, assuming that the flux of Sirius in this range agrees with the flux derived by the theoretical model for the Sirius atmosphere. The flux versus $\lambda$ curve for Sirius shows no trace of $\mathrm{H}_{2} \mathrm{O}$ absorption. Therefore at this height the $\mathrm{H}_{2} \mathrm{O}$ band absorption from the Earth's atmosphere is completely negligible. Then after the instrument has been calibrated using Sirius, several M-type stars have been observed. For example, in the spectrum of Aldebaran and $\mu$ Gem a clear bump is visible at $\lambda=\mathrm{I} \cdot 6 \mu$ and it is explained by the fact that at this wavelength the negative hydrogen ion absorption coefficient has a minimum at this point. For the more advanced spectrum of $\rho$ Persei $\left(\mathrm{M}_{4}\right) \mathrm{H}_{2} \mathrm{O}$ bands due to the atmosphere of the star, appear. $\mathrm{H}_{2} \mathrm{O}$ band intensity appears to depend upon luminosity and to increase with it. An evident luminosity effect is shown by the spectra of $\mu$ Cephei (Mz Ia) and $\mathrm{R}$ Leonis which is a giant of later type. These results show clearly that we must take into account not only the absorption by $\mathrm{H}^{-}$but also by $\mathrm{H}_{2} \mathrm{O}$ in computing models for later type stellar atmospheres. For the future it is planned to use the same telescope to reach a high photographic resolution.

\section{Discussion}

R. Cayrel: Have you evidence for continuous absorption coming from $\mathrm{H}_{2} \mathrm{O}$ in addition to band absorption?

Schwarzschild: I hope that theoretical models are computed which will show which causes of absorption fit better the observations.

\section{INFRA-RED SPECTROSCOPIC OBSERVATIONS OF COOL STARS FROM OUTSIDE}

THE EARTH'S ATMOSPHERE

\section{Yoshio Fujita}

\section{Energy Distribution of Cool Stars in the Infra-red Spectral Region}

It seems very probable that the opacity of cool stars originate from the continuous absorption of $\mathrm{H}^{-}$and $\mathrm{H}_{2}^{-}$, the Rayleigh scattering of $\mathrm{H}$ and $\mathrm{H}_{2}$, and the line absorption of molecular bands. Next it should be mentioned that a blanketing effect due to molecular bands is to be expected in the atmospheres of cool stars. In such circumstances, the spectrophotometric study of these stars in the infra-red region from outside the Earth's atmosphere is most important.

\section{Possibility of Spectroscopic Observations of Some Polyatomic Molecules in the Infra-red Region of Cool Stars}

In research on cool stars, a study of the spectra of polyatomic molecules, especially the study of such molecules as $\mathrm{H}_{2} \mathrm{O}, \mathrm{HCN}, \mathrm{C}_{2} \mathrm{H}$, and $\mathrm{C}_{2} \mathrm{H}_{2}$, etc. which seem to be abundant in these stars, in the infra-red spectral region is important. From the analysis of Y CVn, the length of $\mathbf{L}$ 
the absorption path under the atmospheric pressure becomes $(\mathrm{HCCN}) \sim\left(\mathrm{HCC}_{2} \mathrm{H}_{2}\right) \sim$ $3 \times 10^{2} \mathrm{~m}$. The laboratory experiments show that the main bands of such molecules can be observed if their absorption path is of the order $\mathrm{I} \mathrm{cm}$ and even the bands of higher mode are observable with absorption path shorter than $10^{2} \mathrm{~m}$. This is the reason why spectral lines of polyatomic molecules can be anticipated to have a high intensity in infra-red spectral regions of carbon stars.

\section{Isotopes Abundance Problem in the Infra-red Spectral Region of Carbon Stars}

Our investigation of some carbon stars in the spectral region from $7500 \sim 9000 \AA$ revealed that a quantitative analysis of the $\mathrm{C}^{13}$ isotope might be more easily done if it could be carried out outside the Earth's atmosphere. The amount of the isotopic shift is larger for bands found in the infra-red.

\section{Discussion}

$P$. Boyce: To make best use of available light in the infra-red it will be necessary to use interferometric spectroscopy as done at Lowell Observatory.

\section{PROBLEMS OF ABSOLUTE SPECTROPHOTOMETRY}

\section{F. B. Oke}

To determine as accurately as possible the physical parameters which describe the atmosphere of a star it is important to have observations in all spectral regions. Depending on the temperature of the star, one particular spectral region may be particularly important. Concerning absolute spectrophotometry very accurate ground-based observations, which can be obtained relatively easily, should be obtained in the near future in the wavelength range $\lambda_{3200}$ to $\lambda$ I I 000 or more, so that they will be available when satellite observations are obtained. In order to achieve continuity, satellite instruments should be designed so that observations will overlap those made from the ground.

Photoelectric spectrum scanners are becoming common and relative monochromatic flux measurements can now be readily obtained from $\lambda_{3200}$ to $\lambda_{11} 000$. It is strongly recommended that flux measurements be made with instruments having an intrinsic resolution of a few angstroms, but with bandwidths of approximately $5^{\circ}$ angstroms chosen at selected wavelengths. Such measurements are well defined and independent of any choice of the continuum.

Spectrophotometric standard stars have been selected and carefully measured relative to the primary standard $\alpha$ Lyr. (See Appendix I of the Draft Report, Trans. IAU, I2A, p. 468). Comparison of results from independent observers indicates that there are no systematic errors and the accuracy of the relative monochromatic fluxes is approximately $r \%$ except in the regions $\lambda<3700$ and $\lambda>8500$ where it is about $2 \%$. The most difficult problem is the calibration of the primary standard $\alpha$ Lyr against a laboratory source. The older, rather discordant data have been summarized by Code in a review article. Recent absolute measurements by Chalonge and by Bahner are in excellent agreement. The results for $\alpha \mathrm{Lyr}$, when compared with model atmospheres computed by Mihalas give $T_{\mathrm{e}}=9500^{\circ} \mathrm{K}$ for the continuum. This agrees with the temperature obtained by Hanbury Brown and his collaborators from the measured stellar radius.

Ground-based monochromatic fluxes can be used for several purposes. Among these are the following: ( 1 ) Apart from complications due to reddening and line blanketing, comparisons with model atmospheres yield relative temperatures $\theta_{\mathrm{e}}=5040 / T_{\theta}$ with an accuracy of $0.0 \mathrm{r}$ and in some cases $\log g$ to $\pm 0 \cdot 1$. (2) The fitted model atmospheres can be used to estimate the flux in the ultra-violet for any particular star. (3) For many years to come spectrophotometry of stars fainter than magnitude I I will be done entirely with Earth-based telescopes. 
The assumption that nearby stars are unreddened should be made only with great caution. Absolute spectrophotometry of $\alpha$ Leo indicates that it is reddened by nearly $0^{m_{I}}$ in $B-V$ in spite of its distance of only 75 parsecs.

\section{Discussion}

R. V. Willstrop: I wish to report the results of observations I made at the Radcliffe Observatory in 1962 with a scanner having $50 \AA$ resolution. Relative measurements of 215 stars were made over the range $3300 \AA$ to $6900 \AA$. The tracings have been normalized using observations I made at the Cape of Good Hope (M.N. RAS, 121, I7, I960) in which bright stars were compared with a calibrated lamp through interference filters. The results for the spectral range $4000-6500 \AA$ are in press (Memoirs $R A S$ ); the Cape observations do not cover a sufficiently wide spectral range to normalize the tracings reliably. The results are given at $25 \AA$ intervals in the unit ${ }^{1} 0^{-11}$ ergs $\mathrm{cm}^{-2} \mathrm{sec}^{-1} \mathrm{~A}^{-1}$. Comparisons with results of Code and Oke indicate differences of the order of $1 \%$.

K. Bahner: On the northern hemisphere, there are two independent sets of 'relative' observations done photoelectrically (Oke and Bahner). The agreement is as good as can be expected (for the few stars in common). The 'absolute' observations mentioned in $A p . \mathcal{F}$., r38, 1314, are not as good as can be done, systematic errors being a tricky business. The $U-V$ continuum of $\alpha$ Lyr seems to be lower than Code's compilation indicates.

It would make the discussion easier if observers gave their relative measurements as relative data before converting them to 'absolute' fluxes (which have to be changed each time the standard distribution is improved).

A. B. Underhill: Oke has noted that by fitting observed energy distributions to those predicted for the continuous spectrum by model atmospheres one can obtain estimates of effective temperature and $g$ that are consistent with absolute luminosities and other measured quantities. The results generate a feeling of confidence in model atmospheres for early-type stars. I should like to point out that this feeling of confidence is justified only for the continuous spectrum. The predicted line spectrum from models is usually not closely like that for an earlytype star. Because a model predicts well theoretically weak features $\left(l_{\nu} / \kappa_{\nu}<I\right)$ like the continuum or wings of the hydrogen lines is no reason to assume that the model will predict well the stronger lines such as those used for spectral classification. Here the situation is bad and much work needs to be done to clear up the situation.

$R$. N. Thomas: Just don't be too sure that your inability to predict the observed strong lines means that your model of the outer layers of the atmosphere is incorrect. To you, model means $\mathrm{B}_{\nu}\left[\mathrm{T}_{0}(\tau)\right]$, because you use LTE theories of line formation. So you have the additional possibility that your $T_{\mathrm{e}}(\tau)$ may be completely correct, but it is just your theory of line formation that is wrong. Actually one is sure any LTE theory of line formation is wrong for strong lines.

\section{REMARKS ON TERRESTRIAL OZONE BANDS}

\section{A. Underhill}

The ozone bands begin at about $\lambda_{3400}$ and become increasingly strong towards shorter wavelengths. They may be readily observed on spectrograms of $B$ stars obtained with ground-based equipment, and they are chiefly of nuisance value only. For instance, it is difficult to determine the profile of the important line $\mathrm{He}_{\mathrm{II}} \lambda_{32} \mathrm{O}_{3}$ because of blending with an ill-defined ozone band. A definitive profile for these bands might be obtained by comparing tracings of spectra of the same star obtained from above the ozone layer and from below it. The project is simple in principle but some sophistication would be required, for different spectrographic equipment would be used in each case. It would be necessary to correlate the two sets of measurements 
using a calibration source of known reproducible spectral distribution. This is an example of how observations from above the Earth's atmosphere could be used to assist the solution of the problems given by observations from the ground. A band width of $2 \AA$ would be necessary to obtain the necessary resolution.

\section{SUGGESTIONS FOR FUTURE EXPERIMENTS}

\section{Hack}

Some few examples of the enormous number of problems which can be studied by means of the combination of space and ground observations concern the following classes of objects:

(I) Magnetic stars

(2) Metallic line stars

(3) Close binaries and particularly the peculiar system $\epsilon$ Aurigae

\section{Magnetic Stars}

(a) If the excess of heavy elements is due to nuclear reactions occurring in the atmospheres of the stars, we can expect production of $\gamma$ rays at the surface of these stars. Therefore it should be interesting to attempt to detect $\gamma$ radiation in the region 0.2 to $5 \mathrm{MeV}$, which can be due to fusion of light elements and electron-positron annihilation. $\gamma$ radiation in the region $50-200 \mathrm{MeV}$ (resulting from decay of $\pi$-mesons produced in nuclear interactions with highenergy particles) could be observed from balloons.

(b) Overabundances of $\mathrm{Li}, \mathrm{Be}, \mathrm{B}$ can be expected as the result of spallation process in the atmosphere. Ground observations already indicate an excess of $\mathrm{Be}$ and $\mathrm{Li}$ in some magnetic stars. Therefore it should be interesting to observe the range $3000-1000 \AA$ and $200-170 \AA$ where lines of $\mathrm{Li}$ I, $\mathrm{Li}$ II, Be I, Be Ir, and $\mathrm{B}$ II are present. I and 2 angströms resolution should be necessary.

(c) Due to the presence of strong magnetic fields, it is possible that the continuous spectrum has a non-thermal component due to synchrotron radiation, which could be dominant in the $U V$ region. It is therefore desirable to compare the $U V$ continuous of magnetic stars with the continuous of normal stars of about the same spectral class. ro-20 angströms resolution should be sufficient.

\section{Metallic Line Stars}

The continua of these stars differ from the continua of the normal stars because for the same $B-V$ color they have lower $U-B$ colors. This suggests an excess of metals since the behavior is opposite to that of metal-poor stars. It is possible that the $U V$ continuous presents similar or stronger peculiarities.

\section{e Aurigae}

According to the observations of the last eclipse, there are several evidences that the eclipse is due to an extended envelope surrounding the invisible companion, which should be a hot star (O- or B-type) and should be the ionizing agent of the envelope. Observations in the 3000$900 \AA$ region could give a confirmation about the nature of the companion. A resolution of 50-100 $\AA$ should be sufficient.

\section{Discussion}

Buscombe: Are the temperatures in atmospheres in A stars really hot enough for nuclear processes to form very heavy elements?

Hack: These are not thermal processes. The strong variable magnetic fields can accelerate the particles at energies corresponding to thermal energies for $T \sim 10^{9}$ or $10^{10}{ }^{\circ} \mathrm{K}$. 
A. Przybylski: Surface reactions, no doubt, take place in peculiar A stars since at least at present they seem to be the only explanation of the abnormal abundance of $\mathrm{He}^{3}$; in the atmosphere of 3 Centauri. However, $\mathrm{He}^{3}$ can fairly easily be converted into $\mathrm{He}^{4}$. The fact that $\mathrm{He}^{3}$ can be observed in the spectrum of 3 Cen seems to indicate that only the simplest nuclear reactions can take place on the surface of peculiar A stars. It would not be possible to explain the abnormal composition of HD IOIO6 $_{5}$ as the result of surface reactions. Its spectrum shows only the presence of the rare earths in the atmosphere besides hydrogen, barium and traces of calcium. The available sources of neutrons would not be sufficient to transform the iron peak into the rare earths. Therefore one has to look for another factor which could lead to an abnormal composition of the outer layers of the star. At present only the separation of the elements in the magnetic field seems to be a plausible working hypothesis for this purpose.

\section{REMARKS ON THE NASA PROGRAM}

\section{A. Boggess}

I would like to amplify somewhat Dr Roman's description of the program at Goddard Space Flight Center. We are putting some emphasis on obtaining ultra-violet objective grating spectra on film which is recovered after the rocket flight. We have several camera systems for this purpose, and should be able to reach fourth to fifth magnitude B stars. One of the cameras is an $f / 0.7 \mathrm{Schmidt}$ with a calcium fluoride corrector transparent down to $1300 \AA$. With a 2160 line/mm grating we get a dispersion of about $60 \AA /$ degree, so that we should be able to separate the strong emission lines of the brighter galactic emission nebulae. Finally, to show that this project is well beyond the planning stage, I can report that we have just a few days ago obtained spectra down to $2000 \AA$ of Jupiter and Venus. Our first look at the spectra shows that we obtained about ro $\AA$ resolution and should be able to derive useful information from these photographs.

\section{REMARKS}

\section{Th. Stecher}

Stecher describes instruments composed of four objective gratings proved in laboratory and carried by two rockets. The results concern the observations of the $U V$ spectra of several stars in the region $1200-4400 \AA$ and the comparison of the observed flux curve with that computed by means of atmospheric models. Several of the observed stars are, in one respect or another, peculiar stars. Among them the Ap star $\epsilon \mathrm{UMa}$; the only lines present in the $U V$ spectrum of this star are the $\mathrm{Mg}$ II lines.

\section{CONCLUDING REMARKS}

\section{H. Aller}

It has been shown by the discussion today that not only can space telescopes supply urgently needed data on stellar spectra, but that these instruments are actually doing so now. The guest investigator program mentioned by Miss Roman will make it possible for a large segment of the astronomical community to participate in this exciting field. There is one point to which I would like to call your attention. Just as the greatest advances have been made where optical and radio data have been obtained for the same objects (e.g. Crab nebula, QSRS's, etc.) so will great advances be made when space and ground-based observations can be combined. It is manifestly absurd to do from space what can be done from the ground and future progress will depend on a judicious combination of the two techniques. Just as radio astronomy has placed a far greater burden on optical telescopes than would have existed otherwise, so will space 
astronomy place a still greater burden on ground-based telescopes. At the present time these telescopes are saturated. There is an acute shortage of adequate, large modern instruments and the situation will worsen before it improves.

\section{APPENDICE I. RAPPORT DU COMITE DES CLASSIFICATIONS STELLAIRES}

\section{Report of Meeting, 31 August 1964}

President: W. P. Bidelman.

SeCRETARY: B. E. Westerlund.

The President discussed the proposed formation of a new Commission on 'Spectral Classifications and Multi-Band Colour Indices' (with authorization of this Commission [No. 45] by action of the General Assembly, the present committee ceased to exist). A list of proposed members of the new Commission was read, and others interested in joining it were asked to inform the President.

Among important activities for the new Commission, Bidelman suggested the organization of several colloquia in the next few years. Also, several working groups will no doubt be needed to cover various aspects of this rapidly growing field.

The President then reported briefly on the IAU Symposium on 'Spectral Classification and Multicolor Photometry' held 17-21 August in Saltsjøbaden, Sweden. The Proceedings of the Symposium are being prepared for publication at the Stockholm Observatory.

The need for a new edition, or at least reissue, of the Yerkes Spectral Atlas was discussed at the suggestion of M. K. V. Bappu and others. In answer to a query by N. G. Roman as to whether the original MKK Atlas could still be reproduced in any manner, A. P. Cowley stated that at least three copies had been made at Yerkes last year. Bidelman cautioned that many of the spectral types given in the original Atlas have now been changed. Ch. Fehrenbach mentioned that he has available a complete set of objective-prism plates of the MK standard stars and that he will immediately undertake the publication of a new spectral atlas similar in format to that already published in his article in Volume $5^{\circ}$ of the Handbuch der Physik. The spectrograms were obtained with a $15-\mathrm{cm}$ objective prism, have a dispersion of $80 \AA / \mathrm{mm}$ between $\mathrm{H} \gamma$ and $\mathrm{H} \delta$, and cover the range $\lambda \lambda$ 3900-5000. It is anticipated that this welcome publication will be available very shortly.

The matter of exchange of information on spectral classifications was then brought up. C. O. R. Jaschek stated that his list of about 15000 stars classified on the MK system by various astronomers from slit and objective-prism spectrograms is now in press (photo-offset). This publication will be distributed to all observatories and to members of Commission 45. Bidelman mentioned that his very complete card catalogue is available for general use. L. N. Mavridis has suggested (to Commission 33) that a general stellar information centre should be set up, perhaps in Athens. Information would then be available on punched cards. At present, however, spectroscopic information on individual stars is best obtained by writing to Drs Bidelman or Jaschek.

Several observational programmes of importance were discussed. Fehrenbach stated that his group will publish MK classifications for all stars for which radial velocities are being determined by his method. A list of fields studied or under study will soon appear in the fournal des 
Observateurs. S. W. McCuskey emphasized the desirability of the reclassification of the stars of the G. C. and similar catalogues. He pointed out that often spectral types represent the weakest point in modern statistical studies, in view of recent improvements in proper motions and photometric data. Bidelman proposed the reclassification on the MK system of all of the HD stars, utilizing moderate-dispersion objective-prism plates. It was generally agreed that it would be highly desirable to have plate material available for accurate classification of any group of stars desired, even if such material were not immediately exhaustively discussed.

According to B. Strömgren, all northern stars of types A-Go and brighter than magnitude $7 \cdot 3$ will soon have been observed in the four-colour system. These observations will be extended to a magnitude limit of $8 \cdot 3$ next year.

Miss Roman mentioned that the Committee on Selected Areas (of Commission 33) intends to list all such areas and also other fields where comprehensive spectroscopic or photometric work is under way. This will be done in collaboration with Commission 45 .

\section{Recommendations}

The great importance of having a number of standard stars observed by all of the classification techniques currently in use was emphasized by several speakers. Likewise it would be important to set up several standard fields containing moderately faint stars; Bidelman suggested (among others) centres at $6^{\mathrm{h}}, 0^{\circ}$ and $18^{\mathrm{h}}, 0^{\circ}$.

W. Becker presented three recommendations concerning practice in spectral classification:

(I) I have tried to estimate the number of symbols now in use as parameters in stellar classification. Including broad-band multicolour photometry (but excluding spectral-type symbols), they sum up to approximately 50 or 6o. Most of them are chosen in such a way that they do not assist the memory. The number has a tendency to increase, and the situation is already confusing. It is very difficult to follow discussions or explanations in terms of diagrams in which three or more such symbols are involved. My recommendation is to avoid such symbols as much as possible and to make more use of the wavelengths involved. Even the first two figures of a wavelength are better than any symbol and are generally sufficient. And, of course, Fraunhofer designations are well-known and unambiguous.

(2) As we now know, only the very young objects of our Galaxy reveal the spiral structure. But not all young objects do so. The young open clusters show it well but individual stars of types $\mathrm{O}-\mathrm{B} 2$ do not do so to the same extent. But there are objects among them that do indicate the spiral structure clearly: the stars responsible for $\mathrm{H}$ il regions. I therefore suggest that such stars should be included in luminosity classification programmes. Lists of these stars have been given by Bok, Bester and Wade, Gum, Gaze and Shajn, Sharpless, and others.

(3) There seem to be at least two different kinds of classification systems. To the first kind belong all classifications that are to be interpreted in terms of the physical properties of stars. There is little need for an extended application to stars not used in establishing the classification itself. To the second kind belong all classifications that are to be applied to completely unknown stars. As quite different systems of classification are appropriate to different types of stars, for the classification of an unknown star one must know what classification scheme to apply to it. This means, in other words, that all of these classifications need a 'preclassification' that tells one which particular classification scheme to apply to a particular star.

My suggestion is that the producers of classifications of the second kind should devote some attention to the question of the relevant and requisite preclassification. As an example, take the narrow-band classification developed by Strömgren. As a preclassification in this case, as Strömgren has already mentioned, an ordinary broad-band three-colour photometry is sufficient. 


\section{APPENDICE II. RAPPORT DU GROUPE DE TRAVAIL SUR LES ETOILES Be}

A Hambourg, le groupe de travail pour les étoiles $\mathrm{Be}$ a eu deux réunions. La première a été consacrée à l'exposé des divers programmes existant actuellement et à une discussion sur les spectres de $\zeta$ Tauri, qui a terminé sa phase de contraction et commencé sa phase d'expansion (observation à Meudon le $3 \mathbf{I}$ Juillet 1964 d'un bord d'émission rouge à $\mathrm{H} \beta$ ). On a insisté sur la nécessité d'obtenir, pour certaines étoiles, des spectres avec une dispersion de l'ordre de ro $\AA / \mathrm{mm}$ qui permettraient de mieux suivre les phénomènes dont l'enveloppe est le siège. En particulier, il est important de pouvoir déterminer la progression Balmer. La deuxième réunion a été consacrée à dresser une liste d'étoiles pour lesquelles tous les observateurs d'étoiles Be devraient s'efforcer d'obtenir des spectres à grande dispersion, le plus fréquemment possible, Cette liste a été établie provisoirement comme suit:

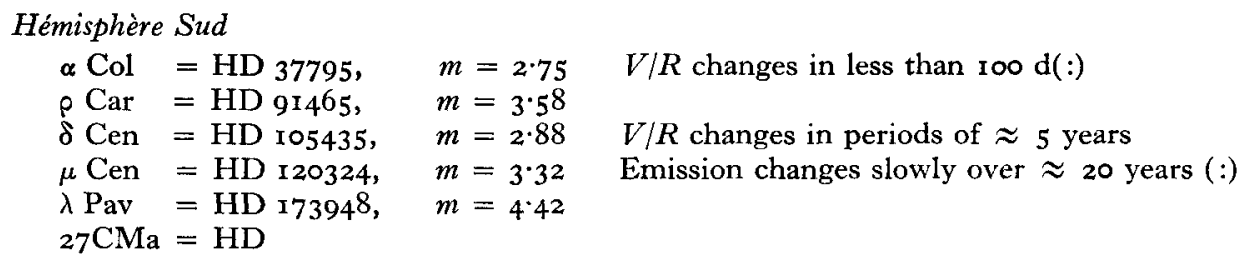

\section{Hémisphère Nord}

\begin{tabular}{|c|c|c|}
\hline HD $5394=\lambda$ Cas & $m=2 \cdot 2$ & \\
\hline HD $_{\text {I05 }}$ 6 $=\varphi$ Per & $m=4 \cdot 2$ & $P=125 \mathrm{j}$ \\
\hline HD $23862=28 \mathrm{Tau}=$ Pleione & $m=5 \cdot 2$ & \\
\hline $\mathrm{HD}_{37202}=\zeta \mathrm{Tau}$ & $m=3.0$ & $V / R$ changes in periods of $\approx 7$ years \\
\hline HD $45725=\beta$ Mon & $m=4.7$ & \\
\hline HD $45726=\beta$ Mon & $m=4 \cdot 6$ & \\
\hline $\mathrm{HD}_{45910}=\mathrm{AX}$ Mon & $m=6 \cdot 7$ & $V / R$ changes metallic lines $P=232.5 \mathrm{j}$ \\
\hline $\mathrm{HD}_{5} \circ \mathrm{I}_{3} 8$ & $m=6.6$ & $V / R$ changes $P \approx 50 \mathrm{j}$ \\
\hline $\mathrm{HD}_{109387}=\kappa$ Dra & $m=3.9$ & \\
\hline $\mathrm{HD}_{\text {I } 42983}=48 \mathrm{Lib}$ & $m=4.7$ & $P \approx I$ mois \\
\hline $\mathrm{HD}_{21257 \mathrm{I}}=\pi \mathrm{Aqr}$ & $m=4 \cdot 6$ & $V / R$ changes $P \approx 4.5$ ans \\
\hline HD 2 I 8393 & $m=6 \cdot 8$ & $P \approx I$ mois \\
\hline $\mathrm{HD}_{\mathrm{I} 93237}=\mathrm{P}$ Cyg & $m=4 \cdot 8$ & raies doubles $P \approx$ I mois? \\
\hline $\mathrm{HD}$ I $48184=\chi \mathrm{Oph}$ & $m=4.9$ & \\
\hline
\end{tabular}

Les besoins en spectres standards ont été examinés. Après discussion sur le procédé de mesure à employer pour la mesure des intensités et des vitesses en émission et absorption, le groupe recommande aux Auteurs de bien spécifier, dans leurs articles, de quelle façon leurs résultats ont été établis.

Le Dr C. Jaschek est chargé de la révision du catalogue du Mount Wilson ainsi que de la continuation de la bibliographie, arrêtée en 1949.

Mme Herman doit diffuser régulièrement un bulletin d'information.

Le groupe a souligné ensuite l'importance que présentent des données photométriques sur le continu. Cette photométrie a été commencée à La Plata par M. Feinstein. Elle doit être entreprise à Toulouse (Dr Pédoussaut) et probablement à McDonald (Station d'Infiernillo). Dr J. Bigay se propose de mesurer l'intensité de l'émission à $\mathrm{H} \beta, \mathrm{H} \gamma$ et $\mathrm{H} \delta$. 